\title{
WAVE PROPAGATION DUE TO AN EMBEDDED SEISMIC SOURCE IN A GRADED HALF-PLANE WITH RELIEF PECULIARITIES. PART II: PARAMETRIC STUDY
}

\author{
I.-K. Fontara, F. Wuttke \\ Institute of Applied Geo-science, \\ Christian-Albrechts-University of Kiel, Germany, \\ e-mails: fontara@gpi.uni-kiel.de, fw@gpi.uni-kiel.de \\ S. PARVANOVA \\ University of Architecture, Civil Engineering and Geodesy, \\ Faculty of Structural Engineering, Structural Mechanics, \\ 1, Hr. Smirnenski Blvd, 1046 Sofia, Bulgaria, \\ e-mail: slp_y@abv.bg \\ P. Dineva \\ Institute of Mechanics, Bulgarian Academy of Sciences, \\ Acad. G. Bonchev St., Bl. 4, 1113 Sofia, Bulgaria, \\ e-mail : dineva@gmail.com
}

[Received 10 February 2015. Accepted 09 March 2015]

\begin{abstract}
The mechanical model and the accompanied computational technique, based on the boundary integral equation method (BIEM) and Green's function for continuously inhomogeneous half-plane were described in the first part of this work. 2D elastodynamic problem for quadratically inhomogeneous and heterogeneous geological area was defined in the first part of our work. The aim of the current second part is to demonstrate the accuracy and the convergence of the proposed computational tool. Furthermore, subsequent extensive parametric study will illustrate,
\end{abstract}

\footnotetext{
${ }^{*}$ Corresponding author e-mail: fontara@gpi.uni-kiel.de

The authors wish to acknowledge support provided through the DFG Grant No. DFG-Wu 496/5-1.

The author P. Dineva acknowledges support by the Bilateral Bulgarian-Greek Project based Personnel Exchange Program between BAS and AUTH, Cooperation Program SCIG.

The author S. Parvanova acknowledges support by the DAAD grant code A/14/02829-Ref. 324 .
} 
that the seismic wave field is a complex result of mutual play of different key factors as free-surface relief, wave characteristics, as frequency and wavelength, seismic source properties, type and characteristics of the material gradient, existence of different type of heterogeneities and their interactions.

KEY WORDS: SH wave, graded half-plane, seismic source, surface relief, site effects, Green's function, BIEM, synthetic seismograms.

\section{Numerical scheme and verification studies}

The numerical scheme for solution of the boundary-value problem defined in Section 2 by the BIEM, using the Green's function for the inhomogeneous half-plane discussed in Section 3 is based on discretization and collocation technique. The good convergence in numerical solution is achieved when the well-known accuracy condition is satisfied: $\lambda \geq 10 l_{B E}$, where $\lambda$ is the wavelength and $l_{B E}$ is the length of the boundary element. In case of constant boundary elements, the jump term in Eq. (4) is $c=0.5$. Two types of integrals appear after discretization: ( $a$ ) regular integrals which are computed by Gauss's integration scheme, for single integrals and Quasi Monte Carlo Method, for double integrals; $(b)$ singular integrals, which are with weak integrable singularity of $\ln r$ type and strong singularity of $1 / r$ type and all are Cauchy principal value integrals. The singular integrals are solved analytically in a small vicinity of the singular point and numerically in the rest of the integration interval. After discretization, collocation and boundary condition satisfaction a system of linear algebraic equations is obtained with respect to the unknown displacements along the boundary $S_{h}$. The described numerical scheme is for solution in frequency domain, when the seismic source is time-harmonic. The solutions in time-domain are obtained by the usage of direct and inverse FFT technique. A source code has been developed using Matlab software [61] and in order to verify its accuracy, several test examples are solved and the results are compared against available analytical and/or numerical results. To the authors' best knowledge, there are no results in literature for quadratically inhomogeneous half-plane with embedded seismic source and with free- and sub-surface relief peculiarities. This is the reason we validate our numerical scheme by test examples for homogeneous case, using the codes developed for inhomogeneous material, but working with zero inhomogeneous coefficient, i.e. $b=0$. We will validate our numerical scheme in the case of time-harmonic seismic source. The solution of this case is given in Lamb [37] in case the seismic source is located at a point along the traction-free surface of a homogeneous half-plane, without any type of heterogeneity. The Lamb's analytical solution can be found also in Achenbach [62]. 
The first test example concerns scenario of the Lamb's problem, see Fig. 2 , where we consider solution of the problem for displacement along the free surface of elastic homogeneous half-plane, due to out of plane time-harmonic line seismic load, acting at point $\mathbf{x}_{\mathbf{0}}$ on the free-surface. Graphically, this solution is illustrated in Yeh et al. [63]. Figure 2a, b presents real and imaginary parts of the normalized free surface displacement for a fixed wave number $k=\left(\omega \sqrt{\rho_{0} / \mu_{0}}\right)=\pi / 2$ of time-harmonic seismic source, located at the point
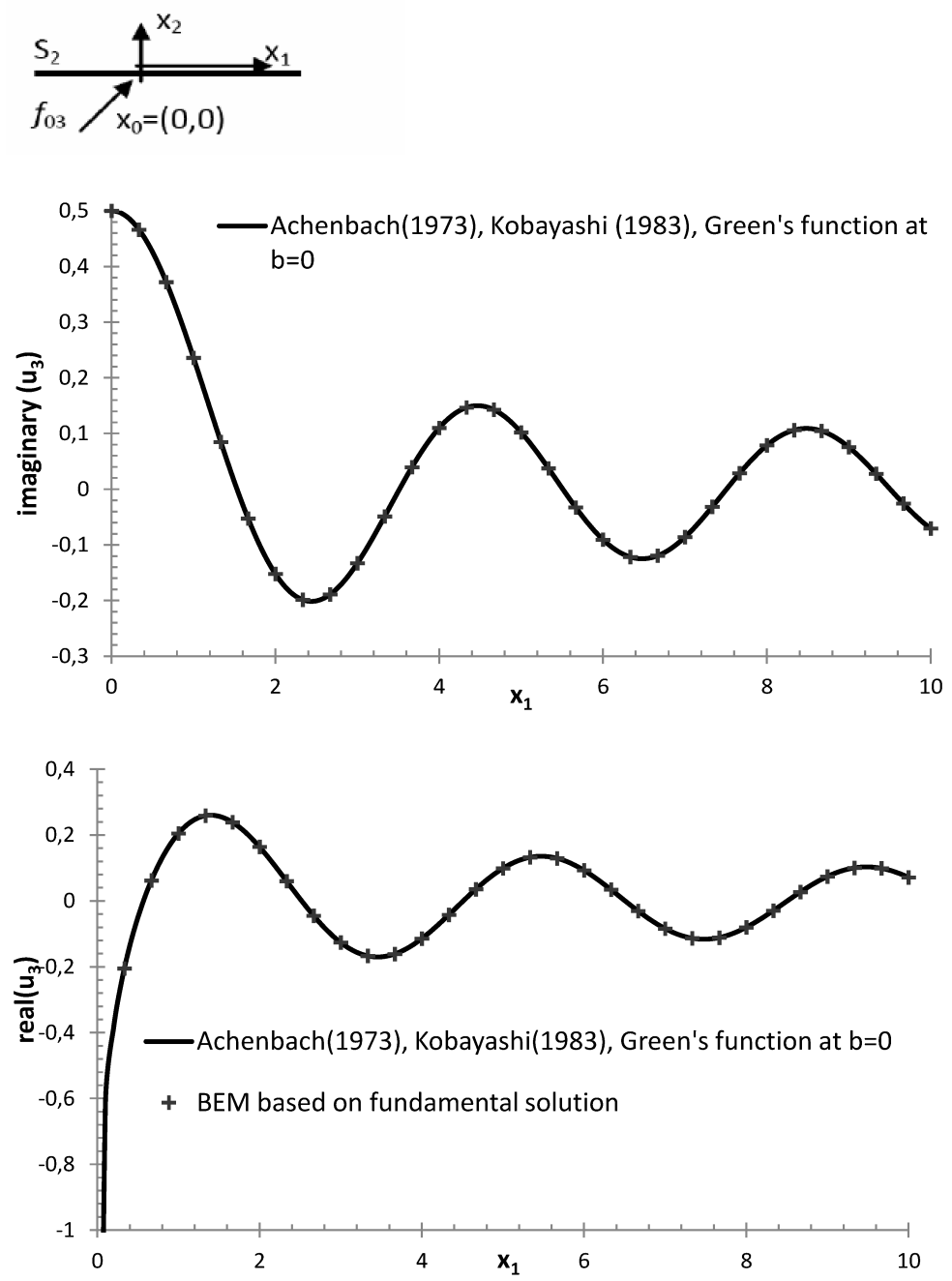

Fig. 2. Comparison with Lamb's solution 
$(0,0)$, along the free surface of a homogeneous half-plane. We compare the results obtained by two different computational tools: (a) analytical solution for homogeneous half-plane expressed by Eq. (8). The corresponding solution for quadratically inhomogeneous case at zero value of the inhomogeneous coefficient $\beta=0$ recovers the solution presented by Eq. (8); (b) numerical solution by direct BEM based on the elastodynamic fundamental solution, for the full plane with the mesh along free surface consisting of 30 quadratic boundary elements. Figure 2 demonstrates that both solutions are indistinguishable. With this test example, we validate our BIEM code, based on the fundamental solution for the full plane. Further, we will validate the proposed here BIEM numerical scheme based on the Green's function, by comparison with the BIEM code, based on the fundamental solution.

We would like to add the following remark. In the mathematical literature, the term "fundamental solution" is used only for the solution of a differential equation with Dirac-function on the right-hand side and the term "Green's function" means the fundamental solution, which additionally satisfies the corresponding boundary condition. The Green's function, if it exists, is unique. The fundamental solution is not unique and it is determined up to a function, which solves the correspondingly homogeneous system.

The second test example considers the wave field in an elastic homogeneous half-plane with material properties $\mu_{0}=123 \cdot 10^{7} \mathrm{~Pa}, \rho_{0}=1850 \mathrm{~N} / \mathrm{m}^{2}$, containing a semi-circle canyon with radius $a$ and subjected to waves radiating from an embedded at point $x_{0}(0,-5 a)$ time-harmonic seismic source. This problem is solved by two different BIEM codes, using fundamental solution and Green's function, correspondingly. Figures 3a-e compare solutions for displacement amplitudes, along the free surface of the half-plane, at different values of the non-dimensional frequency $\eta=2 a / \lambda$. Figure 3 demonstrates the high accuracy of both solutions. The used BEM mesh consists of $24 \mathrm{BE}$ along $S_{\text {can }}$ and 108 along $S_{2}$ constant boundary elements, when use fundamental solution against 14 along $S_{\text {can }}$ constant boundary elements, when use Green's function.

The third test example solves the problem for seismic wave field in an elastic homogeneous half-plane, with material properties $\mu_{0}=123 \cdot 10^{7} \mathrm{~Pa}$, $\rho_{0}=1850 \mathrm{~N} / \mathrm{m}^{2}$, containing an embedded circular cavity with radius $\alpha$, with center coordinates $(\alpha,-0.7 \alpha)$ and also containing a triangle canyon, along free surface, see Fig. 4. The seismic load is presented by incident time-harmonic SH wave with a prescribed frequency $\omega$ and incident angle $\theta$ with respect to the coordinate axis $\mathrm{Ox}_{1}$. Figure 4 compares BIEM results obtained by fundamental solution and Green's function, with solutions obtained in Shah et al. [64] and in Ohtsu et al. [51]. All solutions are almost identical. The used BEM mesh, 

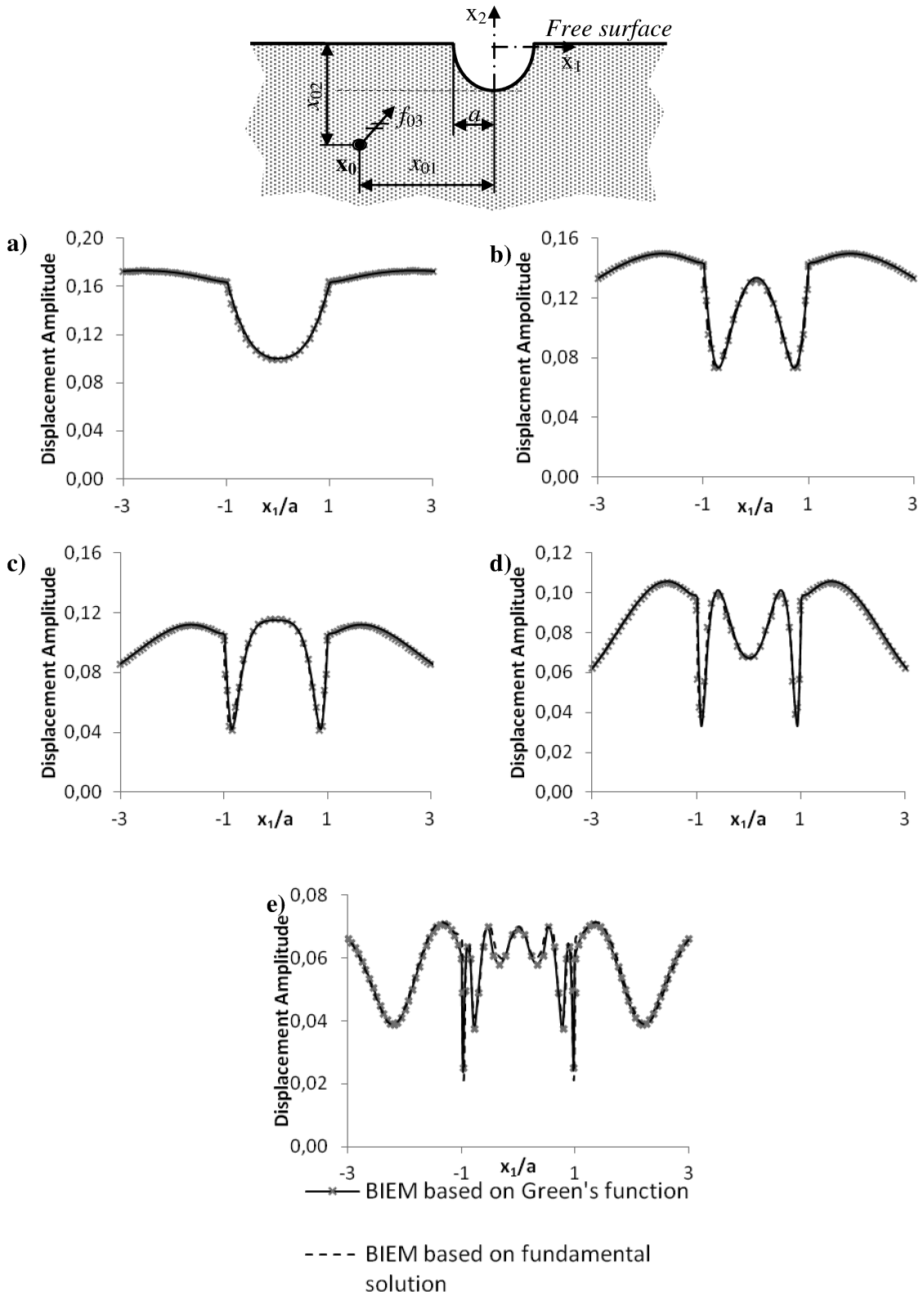

Fig. 3. Displacement amplitude, along the free surface of elastic homogeneous halfplane with a semi-circle canyon, with a radius subjected to waves radiating from an embedded time-harmonic seismic source with non-dimensional frequency $\eta$ at point $(0,-5 a)$ : (a) $\eta=0.25$; (b) $\eta=0.5$; (c) $\eta=0.75$; (d) $\eta=1$; (e) $\eta=2.0$ 

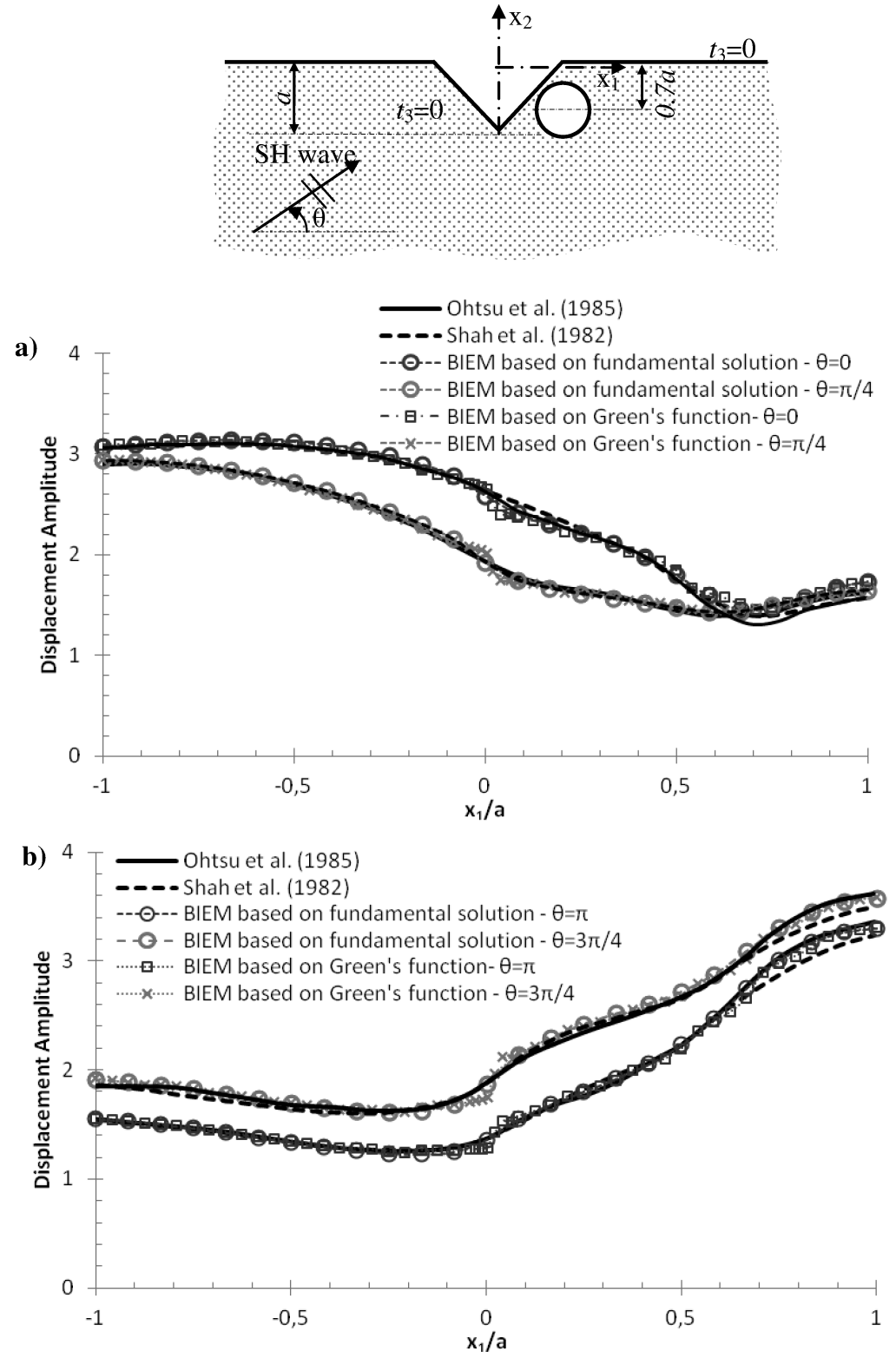

Fig. 4. Displacement amplitudes, along free surface of elastic homogeneous halfplane with triangular canyon and a circle cavity, subjected to incident time-harmonic, with non-dimensional frequency $\eta=0.25$ wave at incident angle : (a) $\theta=0^{\circ} ; 45^{\circ}$; (b) $\theta=135^{\circ} ; 180^{\circ}$. Comparison between solutions in Shah et al. (1982), Ohtsu et al. (1985) and BIEM solutions, obtained by fundamental solution and Green's function 
consists of 24 along $S_{c a n}, 22$ along $S_{c a v}$ and 116 along $S_{2}$ constant boundary elements, when use fundamental solution against 34 constant boundary elements, along $S_{\text {can }} \cup S_{\text {cav }}$, when use Green's function.

The fourth test example compares again BIEM results obtained via fundamental solution and Green's function, for the problem presented in Fig. 5. It considers elastic homogeneous half-plane, without free surface relief but containing a circular cavity with radius $\alpha$ and center coordinates $(0,-5 \alpha)$, subjected to waves radiated from an embedded at point $\mathbf{x}_{\mathbf{0}}(0,-5 a)$ seismic source with a prescribed frequency $\eta=0.25$. Both solutions are fully covered and within plotting accuracy. The used BEM mesh consists of 22 along $S_{c a v}$ and 140 along $S_{2}$ constant boundary elements, when use fundamental solution against 22 along $S_{\text {cav }}$ constant boundary elements, when use Green's function.

The validation results presented in Figs $2-5$ show that the developed numerical scheme and the created software work with a high accuracy and we can continue further with our simulation study.

\section{Parametric study}

The presented in this section numerical simulation reveals convincingly that the seismic wave field is a complex result of mutual internal play of different physical mechanisms, as wave scattering and diffraction, by the free-surface and/or sub-surface relief, taking into consideration the whole seismic scenario, including type, properties and disposition of the seismic source, type, geometry and location of the heterogeneity and also the existence of the material gradient, with respect to the depth. It is introduced the normalized inhomogeneity parameter $\beta=b / 2 \alpha$ of the material inhomogeneity with the following magnitude values $\beta=0$ to $\beta=0.005$. The reference data of the material characteristics are: $\mu_{0}=1.23 .10^{9} \mathrm{~Pa}$ and $\rho_{0}=1.85 .10^{3} \mathrm{~kg} / \mathrm{m}^{3}$ and the magnitude of the applied concentrated force is $f_{03}=10^{9} \mathrm{~N}$. Two locations of the seismic source are considered: $\mathbf{x}_{\mathbf{0 A}}(0,-5 a)$ and $\mathbf{x}_{\mathbf{0} B}(-5 a, 0)$, where $a$ is the radius of the semi-circular canyon (Fig. 3), or the characteristic size of the triangle canyon (Fig. 4).

Figures 6(a), (b), (c), (d) - 7(a), (b), (c), (d) plot the displacement amplitudes along the free surface of the quadratically inhomogeneous half-plane, with a semi-circle canyon, with radius $\alpha$ and embedded time-harmonic seismic source, with normalized frequency $\eta=0.25$ (Figs 6(a), (c) and 7(a), (c)) and $\eta=1.25$ (Figs 6(b), (d) and 7(b), (d)). The seismic source is located at point $(0,-5 a)$ in Fig. 6(a), (b), (c), (d) and at point $(-5 a, 0)$ in Fig. 7(a), (b), (c), (d). Results for different values of normalized inhomogeneity magnitude are shown. 

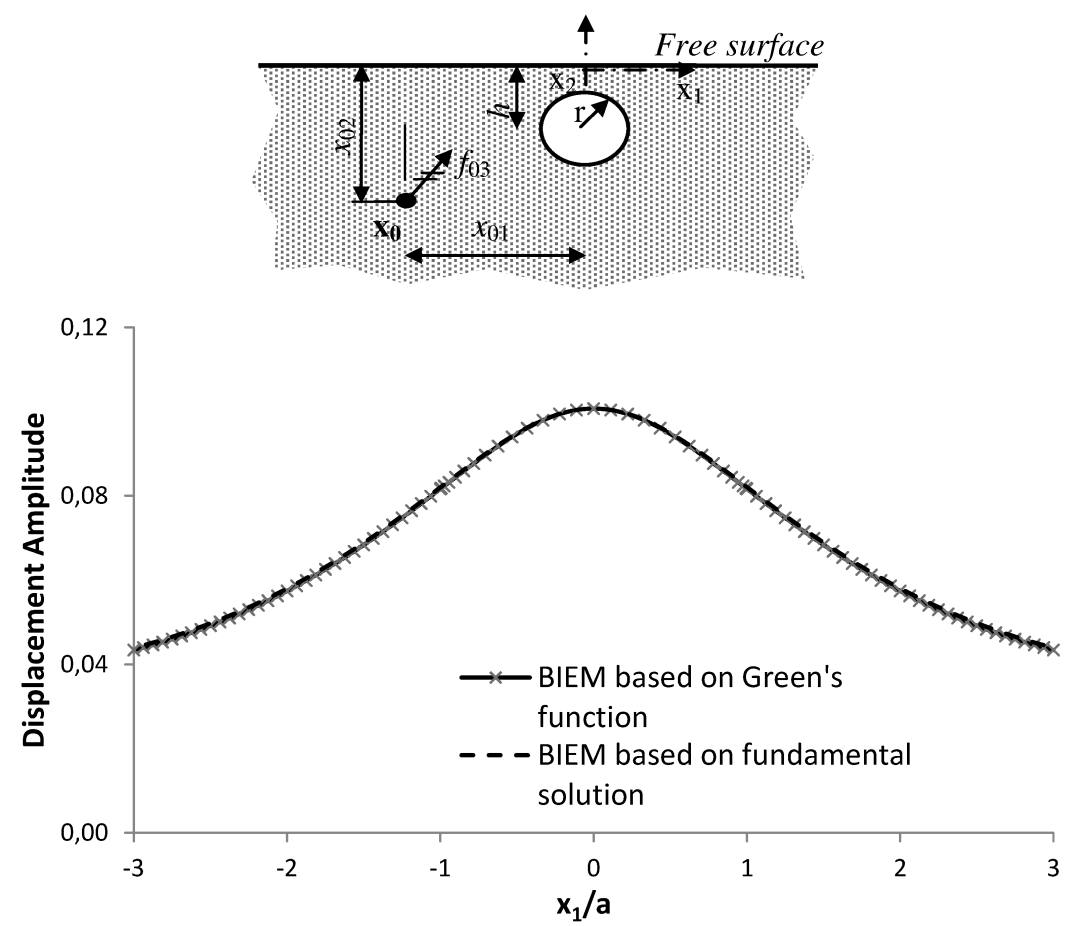

Fig. 5. Displacement amplitudes, along free surface of elastic homogeneous half-plane, with an embedded circle cavity, subjected to waves, radiated by a seismic source, located at point $\mathbf{x}_{\mathbf{0}}(0,-5 a)$, with frequency $\eta=0.25$

Figures 8(a), (b), (c), (d) are analogous, but they concern displacement amplitude distribution, along the free surface of quadratically inhomogeneous half-plane, with a triangle canyon, having the characteristic size $h=a$ and an embedded circular cavity, having the radius $r=0.7 a$ with centre coordinates $x_{1}=a, x_{2}=-0.7 a$, subjected to waves radiating from a seismic source, located at point $(-5 a, 0)$ with frequency $\eta=0.25$ in Fig. 8(a), 8(c) and frequency $\eta=1.25$ in Fig. 8(b), 8(d). In all Figs $6-8$, the displacement amplitude is normalized by $u_{\max }$, where $u_{\max }$ is the maximum value of the response at the free surface of a homogeneous half-plane, without free and sub surface relief for the considered position of the seismic source and frequency value.

Figures 6-8 demonstrate that the seismograms are sensitive: $\left({ }^{*}\right)$ to the seismic source location, compare Figs 6(a), (b) with symmetric wave picture and Figs 7(a), (b), where the wave field is nonsymmetric and is stronger in the zone of source location; $(*)$ to the module of the material gradient, see 

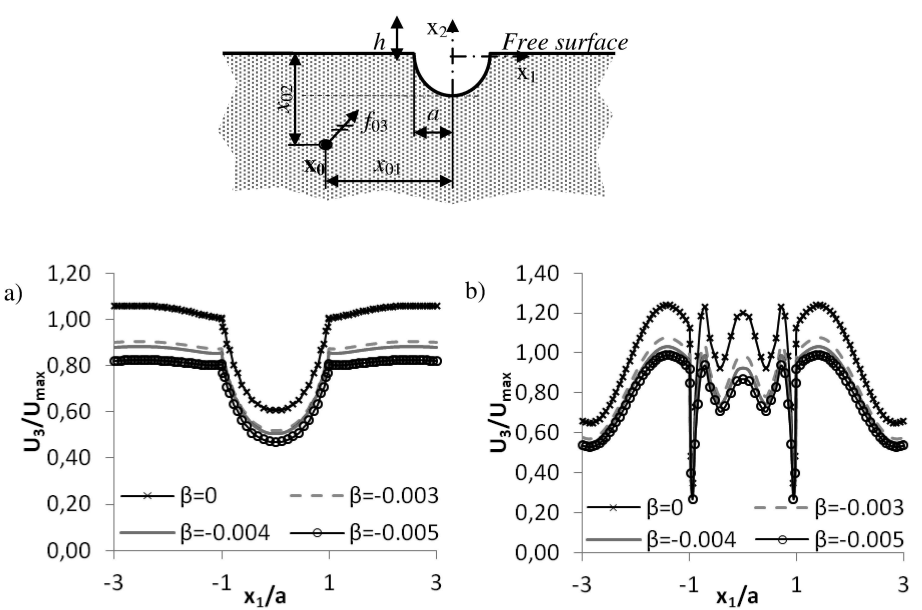

c)

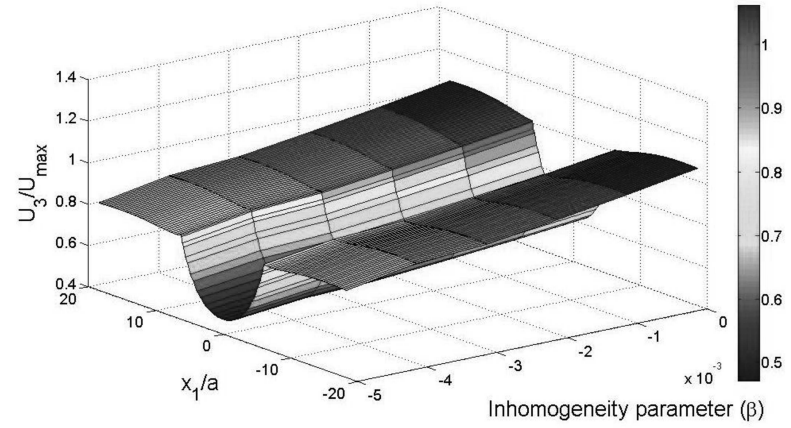

d)

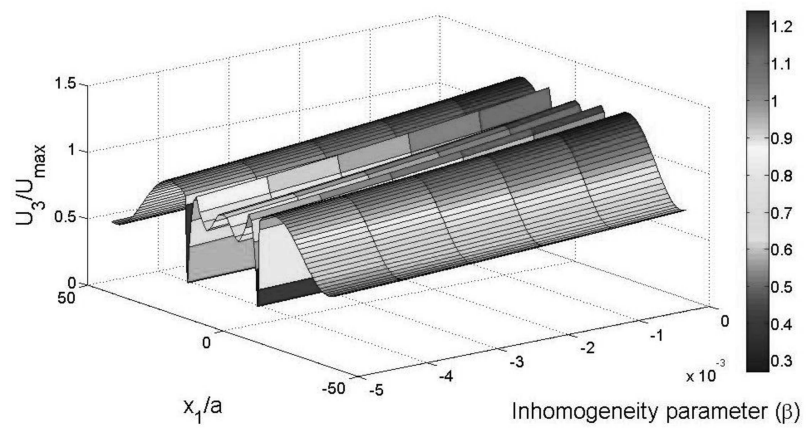

Fig. 6. Normalized displacement amplitude distribution, along the free surface of a quadratically inhomogeneous half-plane, with a semi-circular canyon with radius $a$ and with normalized inhomogeneity parameter $\beta$, subjected to waves, radiating from a seismic source located at point $(0,-5$ a), with non-dimensional frequency $\eta=0.25$, at (a) and (c) and $\eta=1.25$, at (b) and (d) 
a)
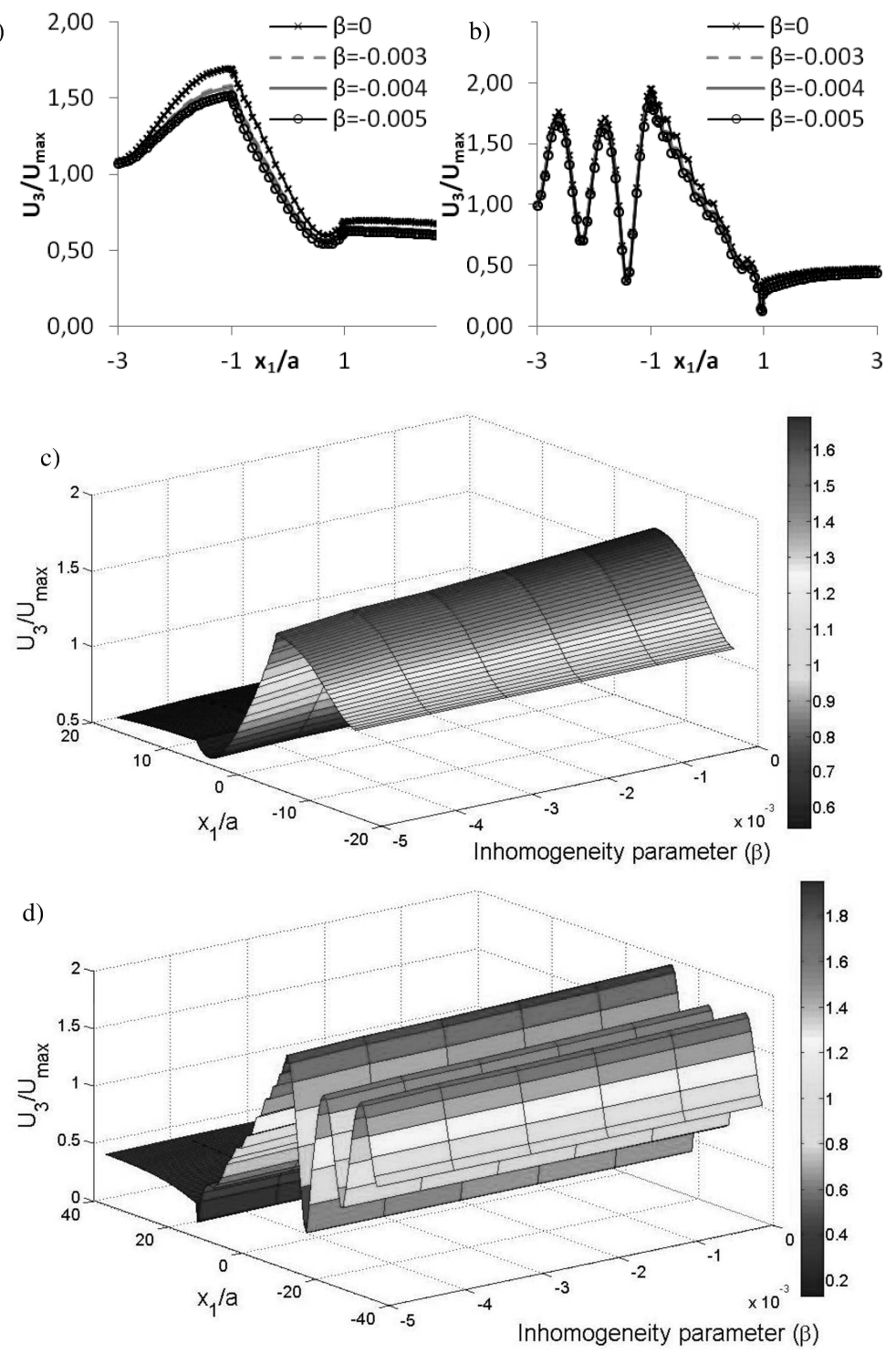

Fig. 7. Displacement amplitude distribution along the free surface of a quadratically inhomogeneous half-plane, with a semi-circular canyon, with radius $a$ and with normalized inhomogeneity parameter $\beta$, subjected to waves, radiating from a seismic source, located at point $(-5 a, 0)$ with non-dimensional frequency $\eta=0.25$, at (a) and (c) and $\eta=1.25$, at (b) and (d) 


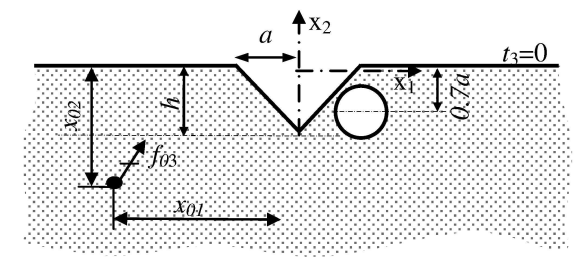

a)

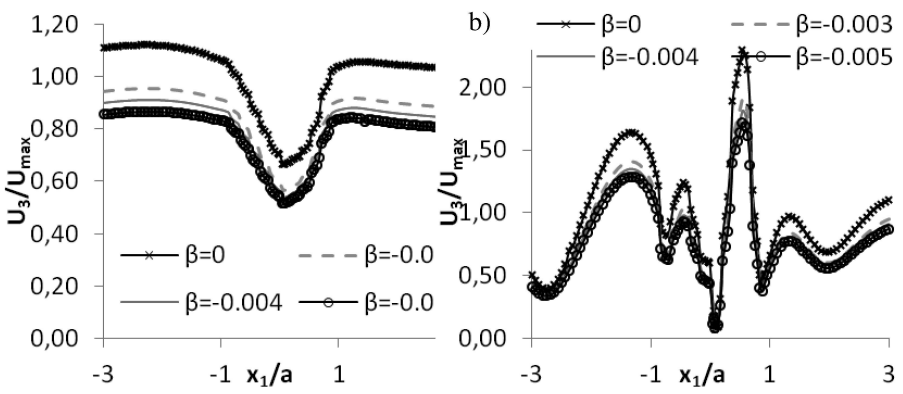

c)
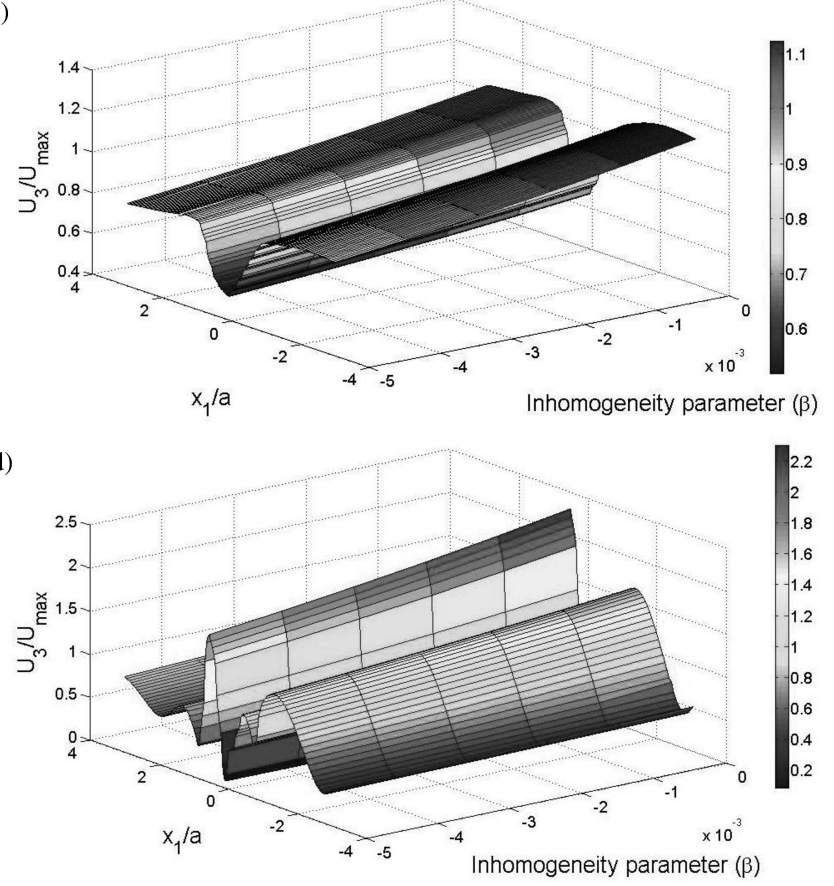

Fig. 8. Displacement amplitude distribution along the free surface of quadratically inhomogeneous half-plane, with a triangle canyon and a circular cavity, with different normalized inhomogeneity parameter $\beta$, subjected to waves, radiating from seismic source, located at point $(-5 a, 0)$ for: (a) $\eta=0.25$; (b) $\eta=1.25$ 
that when the material gradient increases, the displacement amplitude usually decreases; $(*)$ to the existence of sub-surface relief, which can change the seismic field, compare Figs 6(c), (d), 7(c), (d) and 8(c), (d). The strong site effects are visible due to the combining effect of all key factors as material inhomogeneity, lateral inhomogeneity, because of the existence of relief peculiarity, seismic source location and its frequency content.

Results for transient seismic source are shown in Figs 9-11, where the seismic excitation is a vertically, planar Gabor pulse, defined by the following equation:

$$
s(t)=\exp (-\alpha) \cos \left(\omega_{p}\left(t-t_{s}\right)+\psi\right) \quad \alpha=\left(\omega_{p}\left(t-t_{s}\right) / \gamma\right)^{2}
$$

Numerical values for the parameters, appearing in the above equation, are $f_{p}=\omega_{p} / 2 \pi=0.23 ; \gamma=0.15 ; \psi=0 ; t_{s}=0.25 \mathrm{sec}$.

Two boundary-value problems are solved by the proposed BIEM approach for a homogeneous half-plane containing: (a) free surface relief, presented by an elliptic canyon with a prescribed ratio $\kappa=(b / a)=-1.5$ of the semi axes $a=50 \mathrm{~m}$ and $b=\kappa a$, see case (a) in the inserted picture, with the geometry in Fig. 9(a); (b) free surface relief, presented by an elliptic canyon with a prescribed ratio $\kappa=-1.5$ plus a sub-surface relief as a circular cavity with radius $r=20 \mathrm{~m}$ and with center coordinates $(100,-35)$ see case (b) in the inserted picture, with the geometry in Fig. 9(b). The reference data for the material characteristics are $\mu_{0}=10^{10} \mathrm{~Pa}$ and $\rho_{0}=2.5 .10^{3} \mathrm{~kg} / \mathrm{m}^{3}$. The source is located at the point $(0,2 a)$. Figure 9 depicts both time variation and frequency spectrum of the normalized Gabor pulse amplitude.

Figure 10 draws normalized displacement seismograms for receiver sites A (the right rim of the canyon) and B (the bottom of the canyon) in the homogeneous half-plane, with an elliptic canyon. In Fig. 11(a), (b) are presented normalized displacement response spectra for receiver sites $A_{\text {right }}$ (the right rim of the canyon), $A_{\text {left }}$ (left rim of the canyon) and B (the bottom of the canyon) in the following cases: (a) half-plane with elliptic canyon; (b) half-plane with elliptic canyon and a cavity. The normalization is performed by the maximum value of the response at the surface of a homogeneous half-plane, without free and sub surface relief and the same material characteristics, under the same seismic signal.

The simulation results in Figs 10-11 show the sensitivity of the seismic signals to the material inhomogeneity of the wave path, to the type and the location of the seismic source and to the existence of type and shape of any relief peculiarities in the seismic region. The influence of the canyon topographic effects and the existence of buried lined tunnels on the ground motions, recorded 
(a)

Case (a):

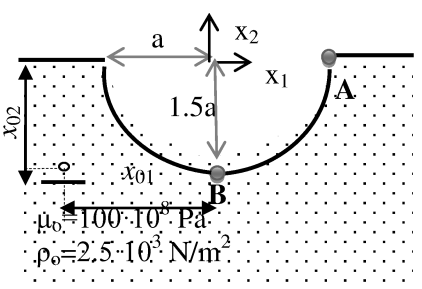

Case (b):

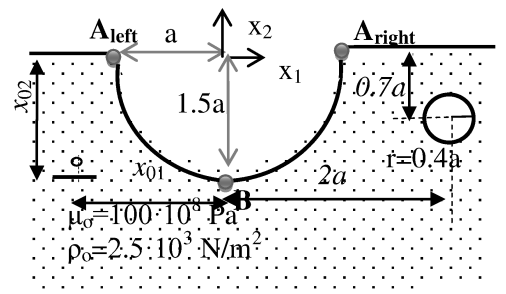

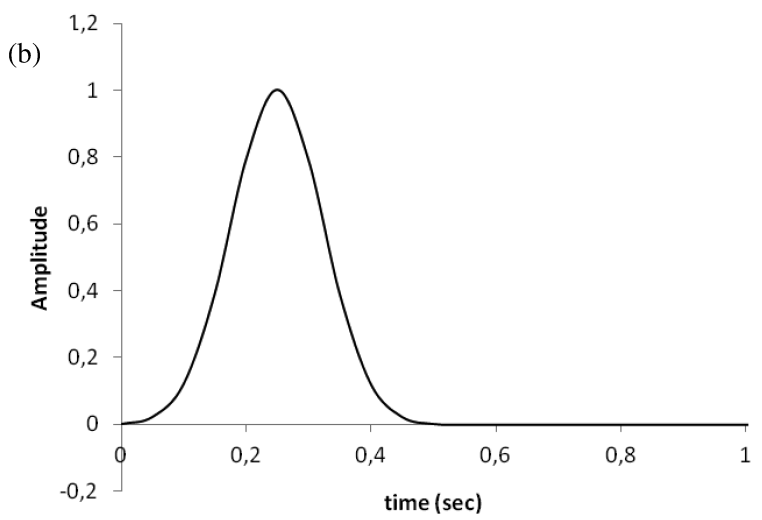

(c)

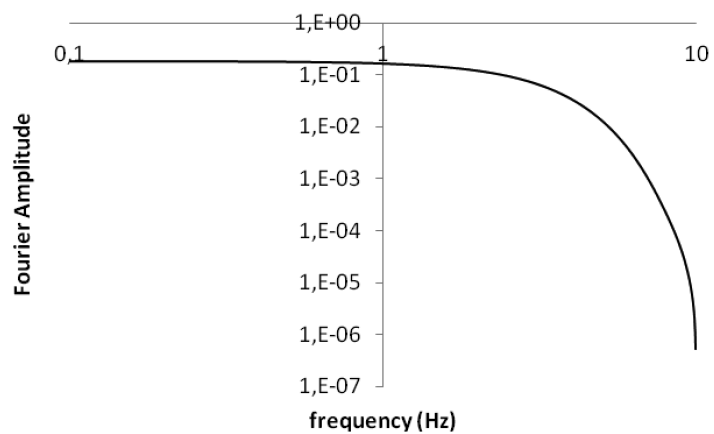

Fig. 9. Time variation and frequency spectrum of the normalized Gabor pulse amplitude

at the free surface is demonstrated. Thus, the presence of local site conditions cannot be ignored and influence the input ground motions used for the design and the analysis of infrastructures. 


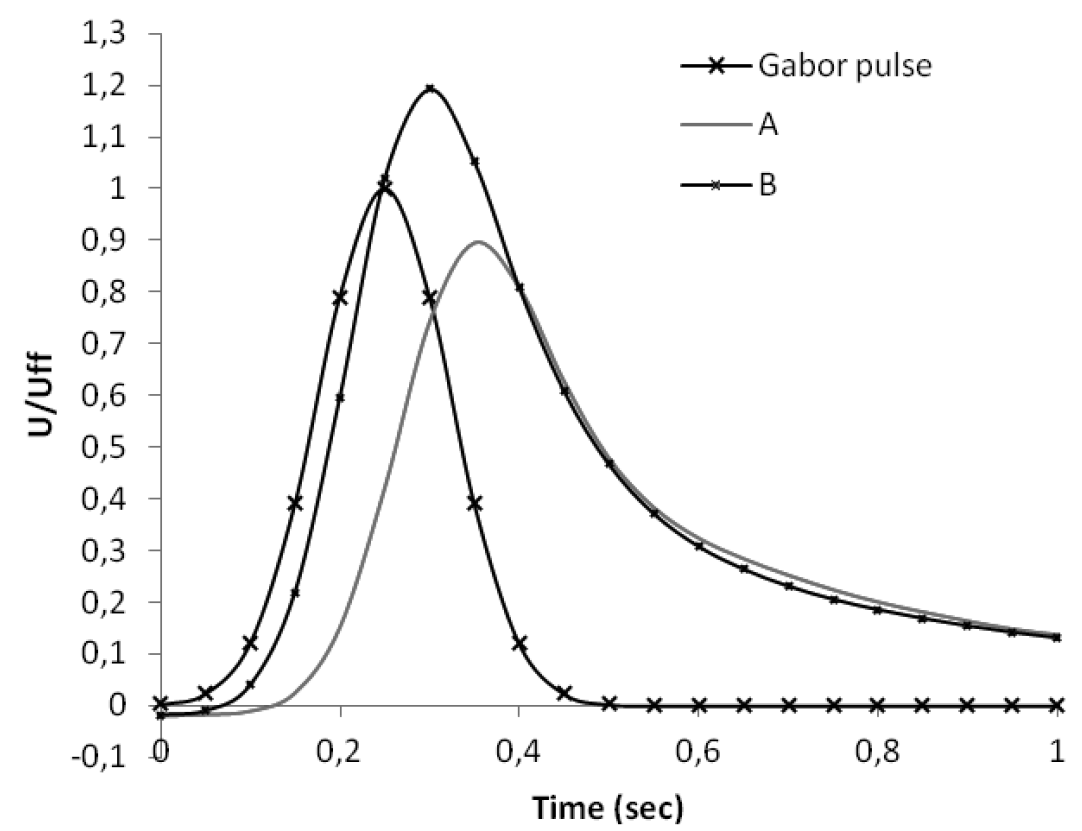

Fig. 10. Normalized displacement seismograms for receiver sites $A_{\text {right }}$ (the right rim of the canyon) and B (the bottom of the canyon) in the (half-plane) with an elliptic canyon

\section{Conclusion}

The anti-plane strain elastodynamic problem in a continuously inhomogeneous with respect to the depth half-plane, with free-surface and sub-surface relief, and under the action of an embedded time-harmonic or transient seismic source is considered here. This problem is solved by BIEM based on the Green's function for a quadratically graded with respect to depth half-plane. The numerical solution is based on the discretization with constant boundary elements, and nodal collocation, along the heterogeneity's boundary only. The software developed for numerical implementation purposes is validated and employed for numerical simulations, so as to demonstrate the sensitivity of the seismic field to the following key factors: type, location and characteristics of the seismic source, scattering and diffraction from the free-surface and sub-surface relief of arbitrary geometry and variable, with the depth mechanical properties of the geological region.

The advantages of the BIE computational technique are as follows: (a) only the boundary of the heterogeneity is discretisized; (b) there is no trun- 
(a)

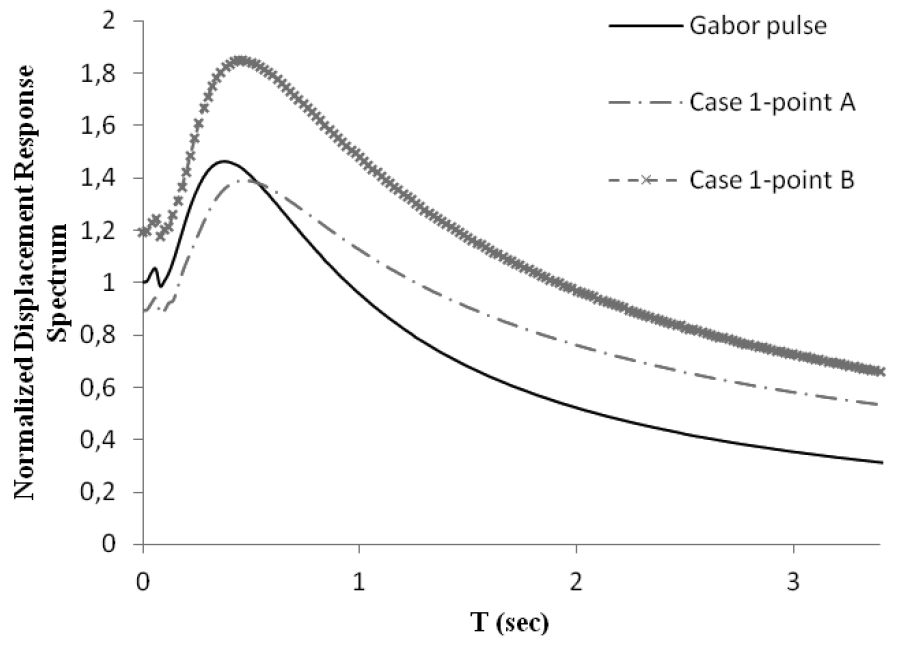

(b)

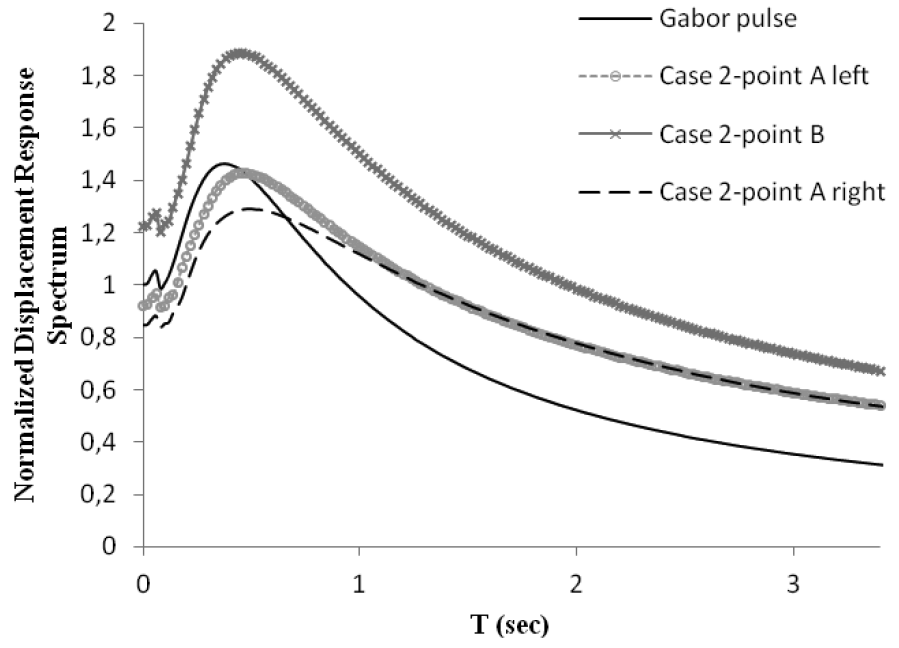

Fig. 11. Normalized displacement response spectra for: (a) receiver sites $A_{\text {right }}$ (the right rim of the canyon) and $\mathrm{B}$ (the bottom of the canyon) in the half-plane with an elliptic canyon; (b) receiver sites Aright (the right rim of the canyon), $A_{\text {left }}$ (the left rim of the canyon) and B (the bottom of the canyon) in the half-plane with an elliptic canyon and a cavity

cation issue, along free surface because the integral is taken only on the finite part of the free surface relief; (c) the mathematical form of the Green's function is not complex nor difficult to evaluate, so it is relatively simple to insert in the BIEM software. The obtained results reveal the potential of the proposed 
BIEM numerical tool to be developed further, for different type of heterogeneities like inclusions, lined tunnels, cracks and soil-structure systems rested in inhomogeneous half-plane.

\section{REFERENCES}

The references are cited in PART I published in vol. 45 (2015), No. 1, pp. 87-98. 